\title{
Visão do médico solicitante frente ao relatório radiológico: uma revisão narrativa
}

\author{
The requesting physician's view of the radiological report: a narrative review
}

La opinión del médico solicitante sobre el informe radiológico: una revisión narrativa

Rebeca Saraiva Bonfim do Nascimento ${ }^{1 *}$, Vitor Ribeiro de Oliveira Santos ${ }^{1}$, Poliana Terra Pires Ribeiro Coelho Caires ${ }^{1}$, Lívya Oliveira Garcia ${ }^{1}$, Laila Pereira Magalhães ${ }^{1}$, Tâmara Campos Xavier Rodeiro', Gabriela Machado Alves ${ }^{1}$, Rafaela Cardozo Mota², Raffaela Oliveira Pinheiro², Ane Caroline Cavalcante Cardoso².

\section{RESUMO}

Objetivo: Realizar revisão narrativa da literatura na tentativa de melhorar a comunicação entre os médicos solicitantes e o radiologista, definindo quais informações devem ser incluídas num relatório radiológico e qual o formato preferido dos solicitantes. Revisão bibliográfica: O laudo radiológico é a principal forma de comunicação dos radiologistas e dos médicos solicitantes. Frequentemente são escritos em texto livre e estudos mostram que isso pode ser um obstáculo para a eficácia no atendimento ao paciente, gerando insatisfação e reclamações dos solicitantes diante da falta de uniformização das informações obtidas. Assim, há uma necessidade de termos padronizados para que esses documentos sejam interpretados com precisão e consistência. Nesse contexto, temos como exemplo a construção da classificação BI-RADS (Breast Imaging Report and Data System) com o objetivo de promover uma padronização dos laudos mamográficos, tornando as recomendações claras para melhor diagnóstico clínico e tratamento. Considerações finais: Visando melhorar a comunicação entre os médicos solicitantes e o radiologista, é necessária a utilização de laudos estruturados na tentativa de evitar registros extensos e sem objetividade. Além disso, deve haver uma melhora significativa quanto à padronização de termos.

Palavras-chave: Laudo radiológico, Padronização, Comunicação.

\begin{abstract}
Objective: To carry out a narrative review of the literature in an attempt to improve communication between the requesting physicians and the radiologist, defining which information should be included in a radiological report and which is the preferred format of the applicants. Bibliographic review: The radiological report is the main form of communication for radiologists and requesting physicians. They are often written in free text and studies show that this can be an obstacle to the effectiveness of patient care, generating dissatisfaction and complaints from the applicants in the face of the lack of uniformity of the information obtained. Thus, there is a need for standardized terms for these documents to be interpreted with precision and consistency. In this context, we have as an example the construction of the BI-RADS classification (Breast Imaging Report and Data System) in order to promote a standardization of mammographic reports, making clear recommendations for better clinical diagnosis and treatment. Final considerations: In order to improve communication between the requesting doctors and the radiologist, it is necessary to use structured reports in an attempt to avoid extensive and objective records. In addition, there should be a significant improvement in terms of standardization of terms.
\end{abstract}

Keywords: Radiological report, Standardization, Communication.

\footnotetext{
${ }^{1}$ Faculdades Santo Agostinho (FASA), Vitória da Conquista - BA. *E-mail: rebecaasaraiva@hotmail.com

${ }^{2}$ Centro Universitário UniFTC (UniFTC), Salvador - BA.
} 


\section{RESUMEN}

Objetivo: Realizar una revisión narrativa de la literatura en un intento de mejorar la comunicación entre el médico solicitante y el radiólogo, definiendo qué información debe incluirse en un informe radiológico y cuál es el formato preferido de los solicitantes. Revisión bibliográfica: El informe radiológico es la principal forma de comunicación para los radiólogos y los médicos solicitantes. Suelen estar redactadas en texto libre y los estudios demuestran que esto puede ser un obstáculo para la efectividad de la atención al paciente, generando insatisfacción y quejas de los solicitantes ante la falta de uniformidad de la información obtenida. Por lo tanto, es necesario contar con términos estandarizados para que estos documentos se interpreten con precisión y coherencia. En este contexto, tenemos como ejemplo la construcción de la clasificación BI-RADS (Breast Imaging Report and Data System) con el fin de promover una estandarización de los informes mamográficos, haciendo recomendaciones claras para un mejor diagnóstico y tratamiento clínico. Consideraciones finales: Para mejorar la comunicación entre el médico solicitante y el radiólogo, es necesario utilizar informes estructurados en un intento de evitar registros extensos y objetivos. Además, debería haber una mejora significativa en términos de estandarización de términos.

Palabras clave: Informe radiológico, Normalización, Comunicación.

\section{INTRODUÇÃO}

Dentre todas as especialidades médicas, a Radiologia é uma das que mais se relacionam com os adventos das tecnologias de informação, pois demanda do profissional de saúde uma integração entre o seu conhecimento técnico-científico, adquirido durante a formação acadêmica, com os mais modernos equipamentos e sistemas operacionais (OLIVEIRA AF, et al., 2014). Com isso, nas últimas décadas os estudos de imagem mostram-se cada vez mais complexos e desafiadores, tanto para o médico que possui as ferramentas necessárias para elaborar o diagnóstico por imagem quanto para aquele que vivencia a prática clínica (SCHWARTZ LH, et al., 2011).

Do ponto de vista universal, ainda não há uma definição única de como os especialistas devem elaborar um laudo dentro do âmbito da imaginologia. Por conseguinte, os médicos radiologistas podem possuir um estilo próprio para a organização do relato ou ainda seguir o formato dos departamentos onde prestam serviço. Nessa perspectiva, é válido ressaltar que diferentes profissionais de saúde podem possuir opiniões divergentes sobre o estilo e a escrita ideal do documento, porém, mesmo respeitando esse posicionamento, a construção de um relatório preciso e que oferece informações pertinentes e úteis para a investigação do quadro clínico do paciente deve ser sempre priorizada (EUROPEAN SOCIETY OF RADIOLOGY, 2011).

O laudo radiológico escrito é a principal forma de comunicação dos radiologistas e dos médicos solicitantes. Um médico solicitante, geralmente, só conhece o radiologista por seus relatórios e esse não sabe quem está recebendo os seus laudos, como eles são avaliados ou o que é esperado pelos médicos (IGNÁCIO FCGR, et al., 2018). Os relatórios são escritos na maioria das vezes utilizando texto livre, entretanto estudos sugerem que tal postura pode ser um obstáculo para a eficácia no atendimento ao paciente, pois eles, por responderem principalmente à questão clínica, acabam gerando reclamações dos solicitantes diante da falta de uniformização das informações obtidas (CAMILO DMR, et al., 2019; BARBOSA F, et al., 2010).

Partindo desse pressuposto, temos que uma parte significativa dos profissionais de saúde apresentam alguma dificuldade em compreender a linguagem técnica do médico especialista em radiologia, sendo necessário, ao menos na conclusão, o uso de termos mais compreensíveis (IGNÁCIO FCGR, et al., 2018). Fardon DF, et al. (2014), exemplificam esse cenário ao relatarem que dentro do âmbito dos estudos de imagem da coluna vertebral existem variados vocábulos para descrever achados normais e patológicos, o que, por sua vez, não contribui para que esses documentos sejam escritos de forma precisa e consistente.

Do ponto de vista histórico, os relatórios de radiologia são rotineiramente escritos em texto livre e de estilo narrativo, permitindo aos radiologistas uma autonomia máxima. Todavia, tal fato também pode levar a uma grande variabilidade e omissão de informações importantes, tornando os relatórios menos úteis e levando a 
uma codificação indefinida. Percebendo esse entrave em sua categoria, surge a classificação Breast Imaging Report and Data System (BI-RADS) com o objetivo de promover uma uniformização dos relatórios mamográficos tornando os achados padronizados e as recomendações claras para melhor diagnóstico clínico e tomada de decisão terapêutica. A especialidade da mama, portanto, torna-se a área pioneira nesse sistema internacional, servindo de exemplo para outras especialidades (PYSARENKO KP, et al., 2017).

Embora o entendimento claro da terminologia nos laudos entre radiologista e médico encaminhador seja essencial para uma compreensão mais adequada do processo-saúde doença, essa compreensão pode ser crítica também para pacientes, familiares, empregadores, seguradoras, juristas e pesquisadores (FARDON DF, et al., 2014). Diante disso, instituições de ensino superior estão cada vez mais inserido o estudo radiológico em sua grade curricular a fim de melhorar a qualidade desses laudos e de interagir diferentes disciplinas por meio da análise dos exames de imagem. Afinal, saber interpretar e escolher o exame complementar de forma adequada são fatores que constituem uma base fundamental que os futuros profissionais de saúde necessitam ter para exercer sua função com excelência (SILVA AF, et al., 2019).

Considerando a relevância desse tema, esse trabalho possui como objetivo realizar uma revisão narrativa da literatura na tentativa de melhorar a comunicação entre os médicos solicitantes e o radiologista, definindo quais informações devem ser incluídas num relatório radiológico e qual o formato preferido dos solicitantes.

\section{REVISÂO BIBLIOGRÁFICA}

\section{O papel do radiologista no cuidado com o paciente}

Os exames de imagem são métodos de diagnóstico auxiliares, solicitados pelo médico que acompanha o paciente e que podem ser feitos através de radiografia, tomografia computadorizada, ressonância magnética ou ultrassonografia. Nesse cenário, o especialista em radiologia e diagnóstico por imagem torna-se responsável pela construção de um relatório sobre os achados que podem ser patológicos ou não. Esse documento deve falar também sobre a técnica que foi usada, os dados do paciente, especificações do aparelho, a região analisada e as possíveis intercorrências. Para mais, é necessário ainda apontar os resultados e suas conclusões de forma fundamentada. Assim, ao final, o relatório é enviado ao médico solicitante que continuará com a investigação clínica do paciente (BARBOSA F, et al., 2010).

À vista disso, percebe-se, portanto, que o laudo radiológico é um resultado direto das informações coletadas durante o exame de imagem. Dessa forma, a descrição deve ser realizada de forma breve e completa, abordando quaisquer questões clínicas específicas em linguagem clara e compreensível. Ainda, durante a sua composição outras qualidades importantes devem ser contempladas, como ser elaborado em formato correto, confiável, sem o emprego de palavras redundantes e consistente. Todavia, a habilidade em analisar as imagens e construir um laudo demanda competências específicas e depende da experiência e conhecimento do radiologista (WALLIS A e MCCOUBRIE P, 2011).

Nesse cenário, os radiologistas desempenham um papel essencial no cuidado com o paciente, pois através da interpretação detalhada da imagem e da comunicação adequada para os demais profissionais de saúde, uma melhor percepção do estado real do indivíduo pode ser contemplada. Apesar de alguns médicos assistentes possam interpretar os estudos de imagem sozinhos, esses documentos quando elaborados por especialistas na área se mostram mais precisos e abrangentes. Com isso, os laudos radiológicos tornam-se ferramentas de extrema importância durante o processo de saúde-doença (CAMILO DMR, et al., 2019).

$\mathrm{O}$ atendimento ao paciente deve acontecer de maneira individualizada e ser realizado com cuidado. Entretanto, considerando a diversidade humana, tanto o médico solicitante quanto o especialista podem se deparar com situações clínicas incomuns, gerando mais um desafio na interação entre esses profissionais. Assim, nessas ocasiões o radiologista pode se guiar a partir de algumas orientações como: iniciar a escrita do laudo destacando a incerteza da sua conclusão em exames com imagens desconhecidas; orientar os colegas qual o melhor método de avaliação, caso receba a solicitação de uma técnica inadequada e deixar claro quando há lacunas em seu conhecimento, podendo oferecer nesse caso interpretações preliminares e posteriormente um adendo após investigação mais profunda (HARTUNG MP, et al., 2020). 
A Medicina não é uma ciência exata e isso além de ser inevitável gera incômodo nos profissionais de saúde. Muitos médicos ao estarem em dúvida de uma condição clínica utilizam em excesso os exames de imagem e isso exige que o radiologista saiba gerenciar o fluxo de informações coletadas, inclusive aquelas em que o resultado é desconhecido. Para isso, o raciocínio Bayesiano é uma ferramenta que auxilia o especialista quando há incerteza, pois, orienta a utilização da probabilidade pré-teste da patologia. Esta nada mais é que a compreensão quantitativa de que a doença possa estar presente naquele determinado achado, considerando tanto os erros intrínsecos quanto as taxas de falso-positivo e falso-negativo da técnica do exame realizada (BRUNO MA, et al., 2017).

Ao se considerar o ambiente hospitalar, percebe-se uma vantagem na comunicação entre esses profissionais, pois, por estarem no mesmo local, os médicos dispõem da facilidade de poder visitar os departamentos de radiologia para discutir laudos e participar de reuniões (WALLIS A e MCCOUBRIE P, 2011). Tal fato contribui também para o crescimento na valorização da relação entre o radiologista e os profissionais de outras especialidades (IGNÁCIO FCGR, et al., 2018). Contudo, esse contato mais direto não acontece dentro de todos os serviços de saúde, logo o alerta de achados graves e até mesmo inesperados necessita ser feito em tempo hábil, conforme atesta Sugimoto K, et al. (2021) ao relatarem que essa falha na comunicação é o principal motivo de negligência médica apontado por seguradoras norte-americanas.

Nessa perspectiva, Farmer $\mathrm{Cl}$, et al. (2020) concluíram que os médicos inseridos dentro do âmbito da Atenção Primária à Saúde exigem um cuidado maior na construção dos relatórios, afinal atuam essencialmente fora do ambiente hospitalar e, portanto, sem interação cotidiana com o especialista. Desse modo, é relatado que esses médicos de referência preferem receber um laudo com indicadores mais claros ao invés de linguagem puramente técnica. Para mais, revelam preferir que o documento também tenha a probabilidade de doença, a relevância clínica dos achados destacados e os tamanhos normais das estruturas anatômicas alteradas.

\section{O laudo radiológico: as vantagens do modelo estruturado}

Há diversas maneiras de escrever um laudo radiológico. Aqueles feitos em prosa permitem ao imaginologista o poder de atribuir a informação que desejar sem qualquer padronização, restrição ou crítica. A descrição dos achados durante o exame é feita de acordo com o conhecimento científico do profissional, da capacidade de síntese, da sua habilidade e experiência adquirida, tornando esse tipo de relatório extremamente subjetivo. Com isso, percebe-se o quanto esse documento é influenciado tanto pelas preferências do autor quanto pelo estilo individual de sua escrita (BARBOSA F, et al., 2010; WALLIS A e MCCOUBRIE P, 2011).

A mudança excessiva na linguagem ou estilo pode minimizar a clareza e dificultar a identificação de informações pertinentes para o atendimento ao paciente (CAMILO DMR, et al., 2019). Por outro lado, os laudos estruturados são elaborados através de formulários em que informações são solicitadas uma a uma via um conjunto de opções pré-determinadas para preenchimento, sendo gravadas em estruturas prédefinidas e restritas de vocábulos. Desta maneira, o médico solicitante aprende e passa a entender as terminologias empregadas por serem restritas e padronizadas, facilitando a compreensão e melhor atenção à saúde do indivíduo. Dessarte, a subjetividade é minimizada, porém é importante destacar que mesmo estruturados nada adianta se o laudo for vago, incompleto ou impreciso (BARBOSA F, et al., 2010).

Esse tipo de formato oferece qualidade e segurança, sendo útil para os residentes por ser um método que contribui no desenvolvimento de padrões de pesquisa organizados. Ademais, permite incluir descobertas positivas e negativas a partir de um feedback consistente e precoce sobre o seu uso apropriado. Dessa forma, os educadores e programas podem utilizar as sugestões a partir desse feedback e sugerir intervenções específicas para aumentar a eficiência durante a construção desse laudo. Logo, percebe-se que a responsabilidade de estabelecer uma comunicação plena extrapola os limites do médico radiologista e solicitante, pois os próprios educadores em radiologia podem influenciar e potencializar a adoção de relatórios estruturados no ambiente de aprendizagem (BURNS J, et al., 2020).

Há uma tendência tecnológica que não permite a incerteza de que o relatório estruturado é uma realidade inevitável dentro da Medicina, pois é um método ágil e evolutivo e seu aperfeiçoamento acontece a partir de estudos analíticos. Assim, as pesquisas sobre esse formato são de extrema importância na prática clínica e 
acadêmica, ainda mais por que alguns profissionais de saúde ainda se mostram resistentes e apegados com as escritas em texto livre. Com isso, torna-se um desafio diário revelar os benefícios, vantagens e o potencial de um laudo estruturado frente aos documentos que promovem técnicas, muitas vezes, obsoletas (ROCHA DM, et al., 2020).

De acordo com Larson DB (2018), um comitê de relatórios estruturados padronizados pode ser criado para estabelecer as prioridades, os padrões e as diretrizes de um laudo. Essa determinação contribui para o estabelecimento de uma estrutura comum em que todos os modelos de relatório são construídos, ajudando a garantir uniformidade, afinal usando esses padrões os membros do comitê podem compor documentos para cada tipo de subespecialidades. Uma vez que os modelos foram criados, editados e publicados, uma forma breve do mesmo processo pode ser usada para manter os relatórios, permitindo uma melhor comunicação aos médicos solicitantes através da aparência concisa e organizada.

Instituições soberanas, como o Colégio Americano e a Sociedade Europeia de Radiologia, recomendam a abordagem estruturada como primeira escolha para a elaboração desses documentos a afim de melhorar a percepção dos resultados dos procedimentos radiológicos. Além disso, tal escrita oferece vantagem sob os textos em prosa por permitir que a informação seja dividida em seções ordenadas (história, indicação, técnica, comparação, achados e impressão), utilizar linguagem padronizada, e ser mais facilmente recuperada ou reutilizada (IGNÁCIO FCGR, et al., 2018). Apesar de nem todos os relatórios aplicarem especificamente esses itens, essa estrutura continua sendo vista como bastante útil e é defendida por muitos autores, pois fornece uma base sólida sobre como produzir laudos adequados (WALLIS A e MCCOUBRIE P, 2011).

Corroborando com esse pensamento, Gassenmaier S, et al. (2017) afirmam que os médicos solicitantes preferem relatórios estruturados e discriminados por permitirem uma retirada mais rápida de informações, pois essas são melhores identificadas com o uso de palavras-chave do que camufladas ou dispersas em todo o texto do laudo. Desse modo, além do benefício que oferece ao percurso clínico do paciente, esse formato também potencializa a construção de pesquisas e estudos retrospectivos. Para mais, foi demonstrado também uma diminuição nos erros de ortografia e semântica, possibilitando um melhor entendimento das informações.

Desse modo, a linguagem deve ser sempre feita de maneira clara, principalmente, para evitar mal entendidos e angústias desnecessárias tanto para o médico de referência quanto para os pacientes, pois estes estão tendo cada vez mais acesso ao resultado dos exames precocemente. Lesões de baixo risco, por exemplo, são frequentemente associadas a um maior nível de preocupação, pois a escrita radiológica pode muitas vezes se apresentar de forma ambígua. Assim, a discrepância entre o que se entende e o real significado proporciona e potencializa um estado ansioso na maioria dos pacientes (ROSENKRANTZ AB, 2017; MITYUL MI, et al., 2018).

Nos últimos anos, houve um empenho para diminuir a variação e linguagem inequívoca nos relatórios que evoluíram com o uso de um formato de texto livre para o uso de modelos estruturados. Especificamente, tem tido um foco em evitar palavras com sinônimos possivelmente confusos e vocábulos que possam representar mais de um significado. Apesar dessas recomendações, ainda não há muitas pesquisas sobre as preferências de idioma e vocabulários usados nos relatórios (ROSS SL, et al., 2017; MITYUL MI, et al., 2018).

Como uma tentativa de alterar esse cenário, em 1995 uma força-tarefa multidisciplinar da Sociedade NorteAmericana da Coluna (NASS) abordou as deficiências nos termos comumente usados e definiu as condições do disco lombar. Ele citou várias documentações do problema e fez recomendações detalhadas para padronização, com o objetivo de promover maior clareza e consistência no uso da terminologia da coluna vertebral. $O$ que vem acontecendo é que algumas palavras e definições usadas para caracterizar um achado não são recomendados como terminologia preferencial, mas é incluído para facilitar a interpretação da imagem e, em alguns casos, o uso é considerado impróprio (FARDON DF, et al., 2014).

Um exemplo disso são as diversas terminologias empregadas para classificar a fissura anular, em que são chamadas "lágrima" e podem ser mal interpretadas por causa da semelhança com outras lágrimas, o que é inapropriado. Ainda, temos o uso do "rasgo" que deve ser desencorajado, devido à preocupação de que essa palavra não fosse compreendida de maneira adequada. Contudo, embora comumente usadas, a confusão 
permanece entre os médicos de diferentes áreas médicas porque não é aceita nas diferentes especialidades. Logo, o esforço em padronizar esquemas de classificação para esses laudos é um posicionamento inerente entre os especialistas (FARDON DF, et al., 2014; YIPING L, et al., 2015).

\section{A Classificação BI-RADS como exemplo a ser seguido}

Alguns esforços já vêm sendo empreendidos na tentativa de melhorar a comunicação entre o radiologista e o médico das demais especialidades. Um dos exemplos mais bem sucedidos e utilizados na prática diária é o BI-RADS, pois o léxico empregado nessa classificação orienta de forma efetiva os profissionais de saúde em sua prática diária. Esse algoritmo não é estático, isto é, pode modificar ao longo do tempo e com o advento da tecnologia e oferece benefícios no rastreamento mamário ao permitir a classificação e estratificação dos achados do paciente (PESCE K, et al., 2019).

O resultado dessa metodologia pode ser direcionado em categorias previamente determinadas e que são numeradas de 0 a 6 , permitindo que os médicos descrevam a mamografia utilizando as mesmas palavras e termos. Logo, isso possibilita uma comunicação com mais precisão e que o acompanhamento das pacientes seja mais efetivo. Diante disso, temos que o BI-RADS 0 é interpretado como achado incompleto, isto é, quando não é capaz de avaliar a mama corretamente e necessita de exames adicionais para comparação. $\mathrm{Na}$ sequência, o BI-RADS 1 é considerado um achado normal da mama, pois não há anormalidades significativas e o tipo 2 é considerado benigno por que não há sinal de câncer e os achados são conhecidos como calcificações benignas, linfonodos na mama ou fibroadenomas calcificados (SILVA VJS, et al., 2019).

O BI-RADS 3 revela achados provavelmente benignos, com a probabilidade de malignidade menor ou igual a $2 \%$, sendo indicado o acompanhamento a cada 6 meses. Por sua vez, o tipo 4 é estabelecido nos achados sem a aparência de malignidade, mas suspeitos, o que justifica a recomendação para biópsia. O BIRADS 5 é altamente suspeito e com uma probabilidade muito alta de malignidade, sendo necessário a realização de biópsia. $O$ aspecto da mama nessa categoria inclui a visualização de massas espiculadas, calcificações lineares finas e/ou massas espiculadas com calcificações pleomórficas. Por fim, o BI-RADS 6 já é uma lesão com diagnóstico de câncer, pois apresenta-se com $100 \%$ de risco e a conduta deve ser feita individualizada, considerando aspectos gerais do tumor (CORRÊA CSL, et al., 2017; SILVA VJS, et al., 2019).

Diante do avanço que essas categorizações permitiram na identificação, tratamento e rastreamento do câncer de mama, percebe-se o quanto outras áreas da Medicina também poderiam se beneficiar com a adoção de um sistema padronizado de informações como o BI-RADS. Afinal, os relatórios tradicionais possuem um formato de texto livre que permite variabilidade excessiva na linguagem, extensão e estilo, podendo minimizar a clareza do documento. Além disso, erros gramaticais, o uso de abreviações e terminologias não usuais representam um desafio para os médicos solicitantes tanto na compreensão do caso clínico e, consequentemente, na sua conduta para com o paciente (GANESHAN D, et al., 2018; SUGIMOTO $\mathrm{K}$, et al., 2021).

Portanto, é perceptivo o quanto o laudo escrito apresenta-se insuficiente na comunicação diagnóstica, principalmente, quando o estado de saúde do paciente é desfavorável. Com isso, a formação do médico durante a residência de radiologia oferece desafios não só na abordagem de relatórios claros e eficientes, mas também na construção da relação médico-paciente que cada vez mais torna-se escassa, pois não há a construção de vínculo, contato visual e empatia (OTONI JC, et al., 2018).

\section{CONSIDERAÇÕES FINAIS}

Observa-se que o laudo radiológico é um dos documentos mais importantes para a Medicina, sendo uma forma de comunicação entre os médicos solicitantes e o radiologista. Por ser um texto livre, há uma falta de padronização e redundância nas conclusões, pois é comum o uso de diversas terminologias para falar de uma mesma doença. Tal fato, por sua vez, prejudica não só a compreensão do profissional de saúde que solicita o exame de imagem, mas também na própria determinação diagnóstica e, consequentemente, na conduta terapêutica do paciente. Dessa forma, é necessário mais incentivo para a utilização de laudos estruturados com o propósito de evitar documentos extensos e sem objetividade. Além disso, torna-se também importante nesse cenário a uniformização de termos, como ocorre com a padronização BI-RADS. 


\section{REFERÊNCIAS}

1. BARBOSA F, et al. Radiological reports: a comparison between the transmission efficiency of information in free text and in structured reports. Clinics, 2010; 65(1): 15-21.

2. BRUNO MA, et al. Communicating Uncertainty in the Radiology Report. American Journal of Roentgenology, 2017; 209(5): 1006-1008.

3. BURNS J, et al. Structured Reports and Radiology Residents: Friends or Foes? Academic Radiology, 2020; 6332(20): $1-5$.

4. CAMILO DMR, et al. Radiology report format preferred by requesting physicians: prospective analysis in a population of physicians at a university hospital. Radiologia Brasileira, 2019; 52(2): 97-103.

5. CORREAA CSL, et al. Rastreamento do câncer de mama em Minas Gerais: avaliação a partir de dados dos sistemas de informações do Sistema Único de Saúde. Epidemiol. Serv. Saúde, 2017; 26(3): 481-492.

6. EUROPEAN SOCIETY OF RADIOLOGY. Boa prática para relatórios radiológicos. 2011. Disponível em: https://www.ncbi.nlm.nih.gov/pmc/articles/PMC3259387/pdf/13244_2011_Article_66.pdf. Acessado em: 02 de março de 2021.

7. FARDON DF, et al. Lumbar Disc Nomenclature: Version 2.0: Recommendations of the Combined Task Forces of the North American Spine Society, the American Society of Spine Radiology and the American Society of Neuroradiology. The Spine Journal, 2014; 14: 2525-2545.

8. FARMER CI, et al. Enhancing clinician and patient understanding of radiology reports: a scoping review of international guidelines. Insights Imaging, 2020; 11(62): 1-10.

9. GANESHAN D, et al. Structured reporting in radiology. Acad Radiol., 2018; 25(1): 66-73.

10. GASSENMAIER S, et al. Structured reporting of MRI of the shoulder - improvement of report quality? European Radiology, 2017; 27(10): 4110-4119.

11. HARTUNG MP, et al. How to create a great radiology report. RadioGraphics, 2020; 40(6): 1658-1670.

12. IGNÁCIO FCGR, et al. Radiology report: what is the opinion of the referring physician? Radiologia Brasileira, 2018; 51(5): 308-312.

13. LARSON DB. Strategies for Implementing a Standardized Structured Radiology Reporting Program. RadioGraphics, 2018; 38(6): 1705-1716.

14. MITYUL MI, et al. Radiology Reporting: Current Practices and an Introduction to Patient-Centered Opportunities for Improvement. American Journal of Roentgenology, 2018; 210(2): 376-385.

15. OLIVEIRA AF, et al. O aprendizado sobre a tecnologia no diagnóstico por imagem. Radiol Bras., $2014 ;$ 47(1): $18-22$.

16. OTONI JC, et al. Comunicação médico-paciente em exames diagnósticos. Radiol Bras., 2018; 51(2): $109-111$.

17. PESCE K, et al. BI-RADS Terminology for Mammography Reports: What Residents Need to Know. RadioGraphics, 2019; 39(2): 319-320.

18. PYSARENKO K, et al. Structured Reporting: A Tool to Improve Reimbursement. Journal of the American College of Radiology, 2017; 14(5): 662-664.

19. ROCHA DM, et al. Evidence of the benefits, advantages and potentialities of the structured radiological report: An integrative review. Artif Intell Med., 2020; 102: e101770.

20. ROSENKRANTZ AB. Differences in perceptions among radiologists, referring physicians, and patients regarding language for incidental findings reporting. AJR Am J Roentgenol, 2017; 208(1): 140-143.

21. ROSS SL, et al. Quantifying Language Before and After Instituting Structured CT Reports. Journal of the American College of Radiology, 2017; 14(11): 1444-1450.

22. SCHWARTZ LH, et al. Improving communication of diagnostic radiology findings through structured reporting. Radiology, 2011; 260(1): 174-181.

23. SILVA AF, et al. Percepção do Estudante de Medicina sobre a Inserção da Radiologia no Ensino de Graduação com Uso de Metodologias Ativas. Rev. bras. educ. med., 2019; 43(2): 95-105.

24. SILVA VJS, et al. BI-RADS Breast Tumor Classification Through Image Mining. Symposium on Knowledge Discovery, Mining and Learning (KDMILE), 2019: 73-80.

25. SUGIMOTO K, et al. Extracting clinical terms from radiology reports with deep learning. Journal of Biomedical Informatics, 2021; 116: e103729.

26. WALLIS A, MCCOUBRIE P. The radiology report-are we getting the message across? Clinical Radiology, 2011; 66(11): 1015-1022.

27. YIPING L, et al. Como devemos classificar a hérnia de disco lombar e a compressão da raiz do nervo? Uma revisão sistemática. Clin Orthop Relat Res., 2015; 473 (6): 1896-902. 